\title{
Recent Advances in Photonic Bandgap Fiber Technology
}

\author{
F. Poletti*, N.V. Wheeler*, N.K. Baddela*, Y. Jung*, J.P. Wooler*, D.R. Gray*, E. Numkam \\ Fokoua*, J.R. Hayes*, R. Slavík*, S.U. Alam*, S.R. Sandoghchi*, V.A.J.M. Sleiffer**, \\ M. Kuschnerov***, M.N. Petrovich*, and D.J. Richardson* \\ * Optoelectronics Research Centre, University of Southampton, Highfield, Southampton, SO17 1BJ, UK \\ ** COBRA institute, Eindhoven University of technology, Eindhoven, The Netherlands \\ *** NSN Optical GmbH, St-Martin-Str. 76, Munich, Germany
}

\begin{abstract}
We review our recent progress in the fabrication, characterization, modeling and splicing of wide transmission bandwidth hollow core photonic bandgap fibers and discuss their modal properties and potential for data transmission.
\end{abstract}

\section{INTRODUCTION}

Hollow core photonic bandgap fibers (PBGFs) represent one of the most radical approaches to address fundamental limits of loss and nonlinearity in transmission fibers [1]. Strong air-guidance with less than $0.1 \%$ of optical power guided in the glass is achieved thanks to a periodic photonic crystal cladding and a large ratio of core diameter to operating wavelength. The fractional overlap with the glass structure not only offers orders-of-magnitude reduction in the optical nonlinearity, but it also allows light propagation at speeds close to the vacuum speed of light (Fig.1). This has appealing advantages for a growing number of ultra-low latency applications [2]. To be able to exploit these characteristics in intra/inter building interconnections for supercomputing/datacenter applications, as well as in metropolitan and potentially ultimately long haul transmissions, a significant further improvement needs to be demonstrated not only in the loss of these fibers, but also in their modal characteristics and inter-modal properties. In this work we discuss our progress in developing PBGFs suitable for high capacity data transmission.

\section{WIDE BANDWIDTH OPERATION THROUGH SURFACE MODE ELIMINATION}

While PBGFs with $<2 \mathrm{~dB} / \mathrm{km}$ loss were demonstrated as far back as in 2004 [3], the early transmission experiments on PBGFs indicated strong modal and

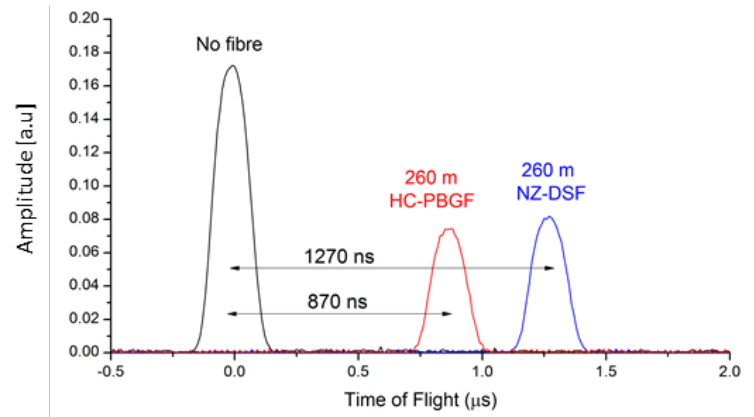

Fig.1. Latency measurement in a PBGF indicating 1.46 times faster light propagation $(1.5 \mu$ s lower latency per $\mathrm{km})$ than in a solid fiber.
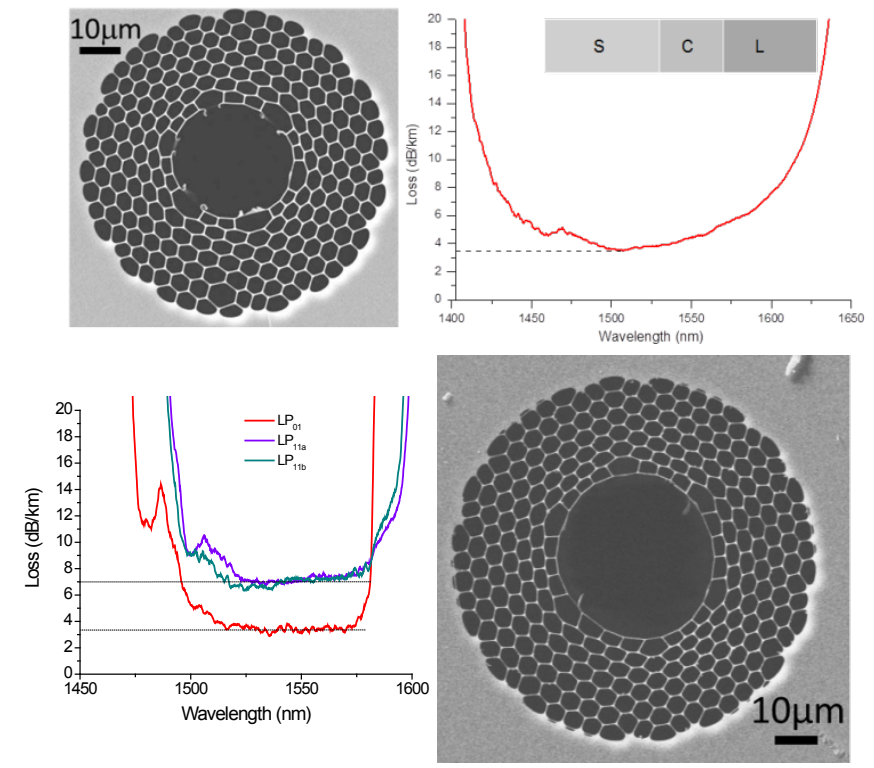

Fig.2. SEM and cutback loss of: (top row) a 19 cell PBGF with a minimum fundamental mode loss of $3.5 \mathrm{~dB} / \mathrm{km}$ and a $3 \mathrm{~dB}$ bandwidth of $160 \mathrm{~nm}$ [4]; (bottom row) a 37 cell PBGF (shown in the same scale) with a loss of 3.3 and $\sim 7 \mathrm{~dB} / \mathrm{km}$ for the $\mathrm{LP}_{01}$ and $\mathrm{LP}_{11}$ modes, respectively.

polarization instabilities which we would now retrospectively attribute to the unsuppressed guidance of surface modes (SMs) at wavelengths close to those used for data transmission. SMs, physically located at an incorrectly terminated or distorted core surround and very susceptible to structural and environmental perturbations, can interact with air-guided core modes causing significant reduction in the useable transmission bandwidth and a severe limitation in the achievable data transmission performances [4].

For this reason, we have focused our recent fabrication efforts on the management of SMs, and developed recipes to produce fibers with a thin and carefully controlled core surround [5]. Although potentially more susceptible to deformations and drawing instabilities, especially in fibers with large core sizes, once perfected, such an approach can lead to the realization of fibers with a combination of low-loss, wide bandwidth and modal qualities that are adequate for data transmission. Recently we have reported two PBGFs with such characteristics: a 19 cell core fiber with a SM-free bandwidth in excess of $150 \mathrm{~nm}$ and loss of $3.5 \mathrm{~dB} / \mathrm{km} \mathrm{[2]}$, and a 37 cell core one with a loss of $3.3 \mathrm{~dB} / \mathrm{km}$ and strong robustness to bending despite its very large mode field diameter of $\sim 23 \mu \mathrm{m}$ [6], see Fig.2. 


\section{Modal ChaRACTERIZATION AND DATA TRANSMISSION EXPERIMENTS}

To reduce the dominating loss mechanism of surface scattering, all low-loss PBGFs must have large cores and do therefore typically support multiple modes. We have studied the modal content of fabricated 19 and 37 cell fibers through both $\mathrm{S}^{2}$ (Fig.3) and time of flight techniques $[2,6]$. Despite a measured loss somewhat higher than the fundamental mode (in agreement with simulations [7]), all higher order modes belonging to the first 4-5 groups were found to be free from anticrossings with SMs throughout the whole C-band and could in principle be used for space division multiplexing (SDM).

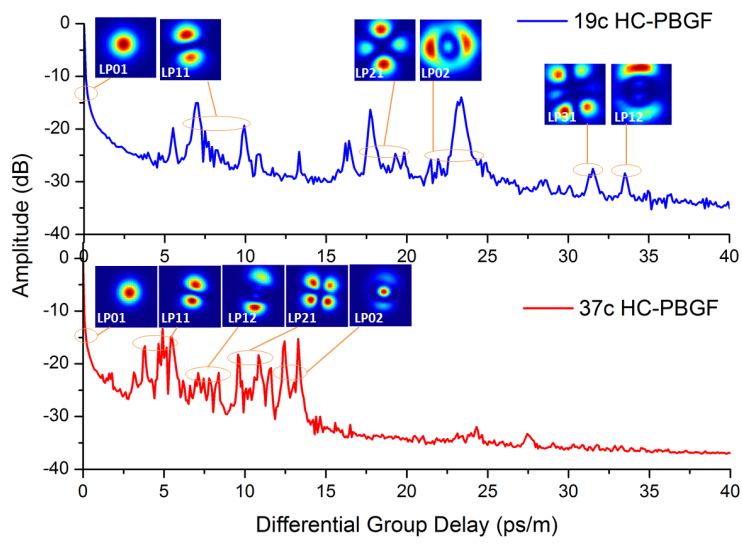

Fig.3. $\mathrm{S}^{2}$ measurement of a 19c (top) and 37c (bottom) PBGF.

Despite guiding tens of vector modes, the intermodal cross-talk in these fibers is sufficiently low to enable an effectively single mode operation over at least the $1 \mathrm{~km}$ fiber lengths we had available for testing. Through selective excitation of the fundamental mode and spatial filtering at the receiver-end we have succeeded in transmitting $37 \times 40 \mathrm{Gbit} / \mathrm{s}$ DWDM on-off keyed channels in the C-band over $250 \mathrm{~m}$ of the 19 cell fiber shown in Fig.1 [2]. The very low power penalties observed in this experiment indicated the possibility to further increase the overall transmitted capacity by exploiting both polarizations, by increasing the number of DWDM channels and by using more spectrally efficient coherent modulation formats. In this way, using 32QAM (2x96x160 Gbit/s/channel/pol.) we have subsequently transmitted down the same fiber up to $30.7 \mathrm{Tbit} / \mathrm{s}$ [8].

Using mode multiplexing techniques it is also possible to excite and transmit cleanly and independently several modes through the PBGF, which opens up the potential for further capacity expansion through MIMO-aided SDM schemes. Using the 3 lower spatial modes (in both polarizations) and 96 DWDM channels modulated with a 16 QAM format, we have recently demonstrated a total data rate of $73.7 \mathrm{~Tb} / \mathrm{s}$ over $310 \mathrm{~m}$ of the 37 cell PBGF shown in Fig.1, which currently stands as the highest data rate ever transmitted through a PBGF [6].

\section{Pbgf Self-Splicing}

A mandatory requirement for PBGFs to be practically useable as transmission fibers is certainly the possibility to be robustly self-spliced with low loss. Although in principle this is a challenging problem to solve as it requires applying sufficient heat to fuse the solid cladding together without causing collapse in the thin microstructured cladding, we have recently demonstrated a splicing recipe to achieve it. Using a sequence of tack, sweep and short arc pulses with a standard fusion splicer we have achieved robust splices with a minimum and average loss of 0.07 and $0.16 \mathrm{~dB}$, respectively (Fig.4), and a negligible impact on the fiber's high order mode content [9]. Subsequent data transmission tests over PBGFs containing splices fabricated with this recipe have shown no significant additional power penalty.
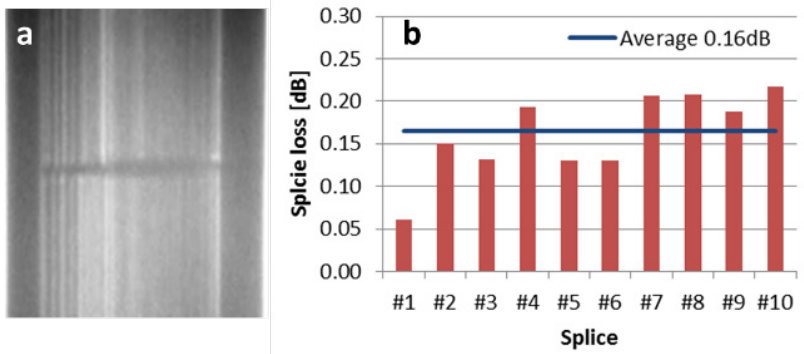

Fig.4. (a) Two PBGFs spliced with the multi-step recipe of Ref. [9] showing no distortion in the microstructure and $\mathrm{a} \sim 10 \mu \mathrm{m}$ residual air gap; (b) Splice loss for 10 different realizations (min: $0.07 \mathrm{~dB}$, average $0.16 \mathrm{~dB})$.

\section{Conclusions}

We have presented some of our latest results in the development of hollow core PBGFs suitable for high capacity data transmission. We have developed recipes to produce fibers with an uninterrupted transmission bandwidth covering the $\mathrm{S}+\mathrm{C}+\mathrm{L}$ bands and realistic prospects of significant further expansion. Once surface modes are eliminated, the air-guided modes of these PBGFs are "well behaved" and their intermodal cross-talk is low enough to allow high capacity single mode transmission. MIMO-assisted SDM can also be achieved to boost-up the total capacity. Finally, we have shown that these fibers can be robustly self-spliced with low-loss using conventional arc-fusion splicers. Although much work remains to be done, we believe that the very encouraging progress achieved over the last two years indicates a bright future for PBGF technology.

\section{ACKNOWLEDGMENT}

This work was supported by the EU 7th Framework Programme under grant agreement 258033 (MODE-GAP) and by the UK EPSRC through grant EP/H02607X/1.

\section{REFERENCES}

[1] R. F. Cregan et al., Science, 285, 1537, (1999)

[2] F. Poletti et al., Nature Photonics, http://dx.doi.org/10.1038/NPHOTON.2013.45 (2013)

[3] B. J. Mangan et al., Proc OFC'04, PDP24

[4] F. Poletti et al., Opt. Express 13, 9115 (2005)

[5] R.Amezcua-Correa et al., Opt.Express 15, 17577 (2007)

[6] Y. Jung et al., Proc OFC'13, PDP5A.3

[7] E. Numkam et al., Opt. Express 20, 20980 (2012)

[8] V. A. J. M. Sleiffer et al., Proc OFC'13, OW1I.5

[9] J. Wooler et al., Proc $O F C^{\prime} 13$, OM3I. 